\title{
Depressão na adolescência: habilidades sociais e variáveis sociodemográficas como fatores de risco/ proteção
}

\section{Depression in adolescence: social skills and socio- demographic variables as risk factors/ protection}

\author{
Depresión en la adolescencia: habilidades sociales y variables \\ sociodemográficas como factores de riesgo/ protección
}

\section{J osiane Rosa Campos*}

Universidade Federal de São Carlos - UFSCar, São Carlos, São Paulo, Brasil

\author{
Almir Del Prette** \\ Universidade Federal de São Carlos - UFSCar, São Carlos, São Paulo, Brasil
}

Zilda Aparecida Pereira Del Prette***

Universidade Federal de São Carlos - UFSCar, São Carlos, São Paulo, Brasil

\begin{abstract}
RESUMO
Habilidades sociais e algumas variáveis sociodemográficas têm sido apontadas como fatores de risco/proteção que podem ser preditivos da depressão em adolescentes. No entanto, ainda não se tem claro quais classes de habilidades e quais variáveis sociodemográficas seriam críticas nessa relação. Este trabalho teve por objetivo investigar quais dessas variáveis podem ser fatores de risco ou proteção da depressão na adolescência, bem como avaliar o poder preditivo de um modelo que inclui essas variáveis. Participaram 642 adolescentes, média de 13 anos, 103 com e 539 sem indicadores de depressão. Os dados, coletados com o Inventário de Habilidades Sociais para Adolescentes (IHSA-Del-Prette), o Inventário de Depressão Infantil (CDI) e o Critério Brasil (CCEB), foram analisados por regressão logística múltipla. Habilidades mais frequentes de empatia e autocontrole bem como idade de 12 anos revelaram-se fatores de proteção; dificuldade nas habilidades de civilidade e sexo feminino mostraram-se fatores de risco. O modelo avaliado apresentou baixo poder preditivo. As implicações dos resultados são discutidas.

Palavras-chaves: habilidades sociais, depressão, adolescentes, fatores de risco/proteção; predição.
\end{abstract}

\section{ABSTRACT}

Social skills and socio-demographic variables have been identified as risk/protective factors that may be predictive of adolescent depression. However, it is not yet clear which classes of social skills and which sociodemographic variables which would be critical in this relation. This study had as aim the investigation of which of these variables could be risk or 
Josiane Rosa Campos, Almir Del Prette, Zilda Aparecida Pereira Del Prette Depressão na adolescência: habilidades sociais e variáveis sociodemográficas como fatores de risco/proteção

protection factors of depression in adolescence, as well as evaluate the predictive power of a model that includes these variables. Participated 642 adolescents (mean age $=13$ years), 103 with and 539 without depression indicators. The data, collected with the Social Skills Inventory for Adolescents (IHSA-Del-Prette), the Children's Depression Inventory (CDI) and the Criterion Brazil (CCEB), were analyzed by multiple logistic regression. Most common skills of empathy and self-control as well as age of 12 years proved protective factors; difficulty in the skills of civility and feminine gender were shown to be risk factors. The model evaluated had low predictive power. The implications of the results are discussed.

Keywords: social skills, depression, adolescence, risk/protection factors, prediction.

\section{RESÚMEN}

Habilidades sociales y algunas variables sociodemográficas se han identificado como factores de riesgo/protección que pueden ser predictivos de la depresión en adolescentes. Sin embargo, todavía no está claro cuáles son las clases de habilidades sociales y las variables sociodemográficas que serían fundamentales en esta relación. Este estudio tuvo como objetivo investigar cuáles de estas variables pueden ser de riesgo o factores protectores de la depresión en la adolescencia, así como la evaluación de la capacidad predictiva de un modelo que incluye estas variables. Participó 642 adolescentes, edad media de 13 años, 103 con y 539, sin indicadores de depresión. Los datos, recopilados con el Inventario de Habilidades Sociales para Adolescentes (IHSA-Del-Prette), Inventario Depresión Infantil (CDI) y el Criterio Brasil (CCEB), se analizaron mediante regresión logística múltiple. La mayoría de las habilidades frecuentes de la empatía y el autocontrol, así como la edad de 12 resultaron factores de protección, dificultad en las habilidades de la civilidad y sexo femenino mostraron ser factores de riesgo. El modelo tenía bajo poder predictivo. Se discuten las implicaciones de los resultados.

Palabras clave: habilidades sociales, depresión, adolescencia, factores de riesgo/protección, predicción.

\section{Introdução}

Ainda que o grupo populacional de adolescentes possa ser considerado saudável, quando comparado a outros grupos, até $20 \%$ dessa população é suscetível às condições negativas à saúde, o que suscita uma preocupação por parte de agências especializadas em saúde pública, como a World Health Organization (2012). Segundo a WHO (2012), dentre os transtornos psicológicos existentes nessa população, o Transtorno Depressivo é retratado na literatura como um dos mais prevalentes. No Brasil, os estudos de rastreamento de sintomas depressivos foram realizados e encontraram uma sintomatologia depressiva variando entre $5 \%$ a $20 \%$ (Bahls, 2002; Reppold \& Hutz, 2003). A WHO (2012) ainda aponta que a diversidade cultural provinda dos dados existentes na literatura dificulta um consenso avaliativo sobre os programas utilizados. Nesse sentido, busca sensibilizar os pesquisadores a produzirem 
conhecimento local, para que as necessidades de saúde mental dos adolescentes sejam suficientemente atendidas.

Os sintomas depressivos em adolescentes são parecidos com os do adulto: agitação ou ansiedade, fadiga, sentimentos de culpa ou inutilidade, dificuldades para tomar decisões, ideação suicida, ruminação, expressões de desamparo, desesperança, insatisfação crônica, retraimento social. Diferentemente do adulto, ao invés de manifestar tristeza, os adolescentes apresentam maior frequência de comportamentos irritadiços e explosivos (Rudolph, 2009; APA, 2005). Os sintomas devem persistir por duas semanas consecutivas, e acompanhados de sofrimento clinicamente relevante ou com prejuízos na área social, ocupacional.

O caráter multicausal e a complexidade do fenômeno depressão são consensos na literatura, isto é, nem sempre existe um fator específico e predeterminante para desencadear, desenvolver ou manter esse quadro. Geralmente, há um grande número de variáveis sociais, psicológicas e biológicas consideradas fatores de risco, tais como: prévio histórico de depressão de um dos pais, viver em famílias consideradas disfuncionais, baixa educação dos pais, eventos estressantes frequentes, pouco suporte social, problemas na escola e de saúde, baixo desempenho acadêmico, ser do sexo feminino e baixo repertório de enfrentamento e de habilidades sociais (Baptista, 1999; Campos, Del Prette \& Del Prette, no prelo).

Embora existam inúmeras teorias explicativas a respeito do fenômeno depressão, como por exemplo, as cognitivas, psicanalíticas e fenomenológicas, no presente estudo compreende-se a depressão sob o prisma de uma visão analitico comportamental. Ferster, Culbertson e Boren (1977) foram pioneiros na análise do problema da depressão sob essa perspectiva. Eles entendiam que os sintomas depressivos seriam decorrentes, em parte, de padrões comportamentais associados a uma história de punição, extinção ou baixas taxas de reforçamento, que gerariam a diminuição da frequência de comportamentos e respostas de fuga e esquiva dos eventos sociais. Dougher e Hackbert (1994/2003) também acrescentam que a persistente punição, falta de reforço ou falha repentina de reforçamento poderiam produzir sentimentos de raiva, frustração, tristeza e cólera, que são respondentes correlatos desses processos.

As pessoas deprimidas, em geral, relatam sentir-se cansadas e apresentam falta de interesse e dificuldade para realizar várias atividades, o que pode gerar baixa taxa de respostas (Lejuez, Hopko, Acierno, Daughters \& Pagoto 2011). Mais recentemente, Boas, Banaco e Borges (2012) sugerem vários motivos para a dificuldade em obter reforçadores ou eliminar e atrasar aversivos: repertório deficitário, falha de controle discriminativo e dificuldade em relação à intensidade (excesso ou insuficiência) da resposta, que não produz a 
consequência esperada. De acordo com Dougher e Hackbert (1994/2003), a falta de interesse pode ser função de operações motivadoras: alguns eventos perdem a função reforçadora e 0 sentimento de prazer associados.

Parte das atividades que são suprimidas e/ou realizadas em baixa frequência refere-se às interações sociais. No caso dos adolescentes deprimidos, estudos têm mostrado que eles relatam muitas dificuldades para desenvolver e manter relacionamentos interpessoais satisfatórios (Garber, 2006). A dificuldade de estabelecer essas relações, por sua vez, pode configurar-se como um dos determinantes e mantenedores dos transtornos depressivos, pois há perda de reforçadores, tais como ajuda, afeto e aprovação social. Nolan, Flynn e Garber (2003) acrescentam, ainda, que frequentemente eles são rejeitados pelos pares de sua convivência. No entanto, como aponta Rudolph (2009) e Segrin (2000), poucos estudos examinaram esta questão, especialmente no início da adolescência. Essa constatação está de acordo com a literatura que aponta para o baixo repertório de habilidades sociais como um dos fatores de risco que contribui para as dificuldades nos relacionamentos interpessoais, presentes nos relatos de adolescentes deprimidos (Segrin, 2000; Gresham, Cook, Crews \& Kern, 2004).

\section{Habilidades Sociais e depressão}

As habilidades sociais são definidas como classes de comportamentos sociais presentes no repertório do indivíduo que possuem alta probabilidade de produzir consequências reforçadoras para 0 indivíduo e para as demais pessoas (Del Prette \& Del Prette, 2010/2012) e que são requeridas para um desempenho socialmente competente (Del Prette \& Del Prette, 2009). A competência social, por sua vez, é um construto avaliativo do desempenho em uma tarefa de interação social que requer articulação de várias habilidades sociais de modo a produzir melhor efeito no sentido de atingir objetivos, melhorar/manter a autoestima dos envolvidos, equilibrar reforçadores e manter/ampliar os direitos humanos (Del Prette \& Del Prette, 2001).

Para a população adolescente, Del Prette e Del Prette (2009) identificaram empiricamente no contexto brasileiro seis classes de habilidades sociais de grande importância: autocontrole, civilidade, empatia, assertividade, abordagem afetiva e desenvoltura social. Por definição, estas classes de habilidades sociais, quando presentes no repertório, podem auxiliar os adolescentes a apresentar desempenhos socialmente competentes, ou seja, que levem a maior satisfação e a relações sociais satisfatórias. Para Segrin e Flora (2000), um indivíduo que apresenta baixo repertório de habilidades 
sociais pode apresentar vulnerabilidade para desenvolver transtornos psicológicos, entre eles a depressão, ao passo que apresentar bom repertório de habilidades sociais facilita o enfrentamento de eventos estressantes que, de maneira geral, funcionam como gatilhos para o desenvolvimento de transtornos depressivos.

O início da adolescência parece ser um momento em que os contextos interpessoais vivenciados pelos adolescentes assumem essencial importância, uma vez que os relacionamentos com os pais se modificam, adquirindo um padrão bastante diferentes dos da infância. Além disso, novas formas são exigidas para se relacionar com os outros adultos. Esta é, também, a etapa de estabelecimentos dos primeiros relacionamentos afetivos/sexuais (Novak \& Pelaez, 2004). Pode-se supor que estes momentos são críticos para a expansão de contatos sociais, o que requer habilidades sociais novas para prover redes sociais, resolver problemas diários e tomar decisão com maior autonomia. Paralelamente, é provável que uma parcela dos adolescentes possa estar exposta a condições estressantes e de risco para o aparecimento de transtornos depressivos (Hammen, 2009), tais como facilidade de obtenção de drogas (cigarros, bebidas alcoólicas, drogas ilícitas), dificuldade para realizar sexo com segurança, como sinaliza o Instituto Brasileiro de Geografia e Estatística (IBGE, 2009), dificuldade de relacionamentos com os pares e afetivos (Davila, 2008) e pais que apresentam práticas parentais inadequadas (Soenens, Park, Vansteenkiste, \& Mouratidis 2012).

Tanto as demandas interpessoais próprias dos adolescentes, como as condições e comportamentos de risco, também prováveis nessa etapa podem requerer um repertório elaborado de habilidades sociais. Em ambos os casos, as habilidades sociais podem se configurar como um fator de proteção para um desenvolvimento saudável e com menor chance de apresentar transtornos. E, em sentido oposto, os déficits seriam fator adicional de risco.

\section{Depressão, habilidade sociais e fatores preditivos: relatos de pesquisas}

A literatura mostra estudos que investigaram relações entre habilidades sociais e transtornos psicológicos na adolescência, tanto de natureza correlacional como preditiva. Em estudos correlacionais, por exemplo, Del Prette, Rocha, Silvares e Del Prette (2012), encontraram que indicadores de depressão estavam significativamente correlacionados com dificuldade em habilidades sociais, tanto no escore geral como nas classes específicas de assertividade e autocontrole. Em outros estudos, as habilidades sociais e/ou competência social foram identificadas como variáveis 
preditivas de depressão na adolescência (Ward, Sylva, \& Gresham, 2010; Ross, Shochet, \& Bellair, 2010; Lee, Hankin, \& Mermelstein, 2010; Nilsen, Karevold, Roysamb, Gustavson, \& Mathiesen, 2013). Nesses estudos, verificou-se predição baixa ou moderada entre baixo repertório de habilidades sociais e indicadores de depressão. Porém, tal repertório era avaliado de maneira geral, não sendo especificadas quais classes poderiam ser preditivas à sintomatologia depressiva na adolescência. De todo modo, estudos de predição, especialmente focalizados nas classes de habilidades sociais enquanto possíveis preditores, não foram encontrados no contexto brasileiro.

\section{Habilidades sociais e variáveis sociodemográficas}

Além das habilidades sociais, algumas variáveis sociodemográficas (sexo feminino, idade e baixo status socioeconômico) são apontadas na literatura (Dimidjian, Barrera, Martell, Muñoz, \& Lewinsohn, 2011), como possíveis fatores de vulnerabilidade ao aparecimento de transtornos depressivos. Considerando que tais variáveis podem ser associadas aos sintomas depressivos, cada uma delas é abordada a seguir.

Status socioeconômico. Alguns estudos internacionais indicam que baixo status socioeconômico está relacionado negativamente ao desenovolvimento de sintomas depressivos em adolescentes (Fortenberry, 2003; Wight, Botticello, \& Aneshensel, 2006). Como apontam Mash e Graham (2005), o impacto das desvantagens econômicas deriva-se do fato de que é uma variável composta que inclui muitas outras influências negativas como, baixa escolaridade, baixo nível de emprego, recursos limitados e exposição à violência fora e dentro da residência. No contexto brasileiro, foi encontrado somente o estudo de Avanci, Assis e Oliveira (2008) que investigou o papel preditor do status socioeconômico sobre a depressão em adolescentes de 11 a 19 anos de idade e o resultado encontrado não confirmou esse poder preditivo, ainda que tenha tido amostra heterogênea em termos de classes sociais.

Idade. Um dos achados mais consistentes é que a prevalência dos transtornos depressivos aumenta com a idade (Méndez, Olivares, \& Ros, 2005). Rudolph (2009) aponta que os transtornos se iniciam a partir dos $12-15$ anos e continuam a aumentar entre os 15-18 anos (Galambos, Leadbeater, \& Barker, 2004). De acordo com Kazdin, Kraemer, Kessler, Kupfer, \& Offord (1997), a idade é uma variável que pode demarcar mudanças na vida do adolescente. Essas mudanças, por sua vez, podem ser um fator de estresse (Moraes \& Rolim, 2012) a exigir novas respostas adaptativas. Nesse sentido, 
parece ser importante incluir esta variável nos estudos de predição, pois esse dado, bastante claro quanto à indicação de depressão, carece ainda de investigações para diferentes faixas etárias.

Sexo/Gênero. Outro achado bastante consistente é referente ao sexo. Os transtornos depressivos incidem numa proporção de duas meninas para um menino (APA, 2005). Como aponta Garber (2006), o sexo não é considerado uma variável passível de mudanças, mas pode influenciar fatores de risco proximais, como as diferenças de resposta ao estresse e os níveis dos neurotransmissores.

Como assinalam Kosterman et al. (2010), o investimento de pesquisas sobre os fatores de risco e proteção envolvidos na indicação de depressão na adolescência, pode ser muito útil para programas preventivos e de promoção de saúde. Considerando a importância de identificar possíveis fatores protetores e de risco relacionados à indicação de depressão na adolescência, e de suprir as lacunas da literatura sobre isso, o presente estudo teve por objetivo investigar quais habilidades sociais e variáveis sociodemográficas podem ser fatores de risco ou proteção da depressão na adolescência, bem como avaliar o poder preditivo de um modelo que inclui essas variáveis, utilizando a regressão logística múltipla.

A Figura 1 apresenta o modelo que reúne variáveis documentadas na literatura (linhas pretas contínuas), algumas delas retestadas neste estudo (em negrito), bem como as classes de habilidades sociais (tracejadas), nos indicadores de frequência (F) e dificuldade (D), enquanto possíveis preditores da depressão na adolescência, ainda não suficientemente investigados. Ressalta-se que o presente estudo foi baseado em indicadores e não em diagnóstico de depressão.

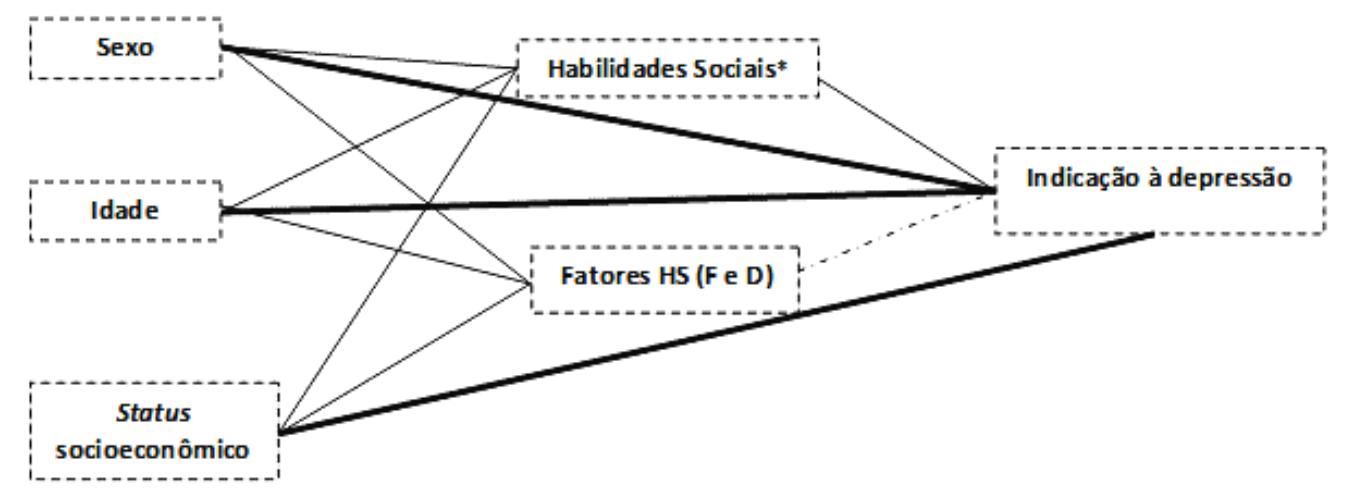

Figura 1. Modelo de relações a serem testadas neste estudo: variáveis sociodemográficas (sexo, idade, status socioeconômico) e os seis fatores de habilidades sociais (HS), nos indicadores de frequência (F) e dificuldade (D) como possíveis preditores de depressão em adolescentes.

\section{Método}




\section{Participantes}

A amostra foi composta por 642 adolescentes de três escolas públicas municipais de uma cidade do interior de São Paulo, de aproximadamente 620 mil habitantes. A coleta de dados foi autorizada pela Secretária Municipal de Educação da cidade e foi selecionada por conveniência. Dessa amostra 103 adolescentes apresentavam indicadores de depressão (Grupo Subclínico) e 539 adolescentes sem indicadores de depressão (Grupo Não Clínico). A Tabela 1 descreve as características sociodemográficas da amostra.

Tabela 1. Características sociodemográficas das amostras do grupo subclínico e não clínico em termos de frequência absoluta (FA) e relativa (FR).

\begin{tabular}{cccccc}
\hline \multirow{2}{*}{ Variáveis } & Níveis & \multicolumn{2}{c}{ Grupo Subclínico } & \multicolumn{2}{c}{ Grupo Não Clínico } \\
& FA & FR & FA & FR \\
\hline Sexo & Ferrinino & 75 & 72,8 & 250 & 46,4 \\
Idade & Masculino & 28 & 27,2 & 289 & 53,6 \\
& 12 anos & 30 & 29,1 & 101 & 18,7 \\
& 13 anos & 39 & 37,9 & 210 & 39,0 \\
Média (DP) & 14 anos & 34 & 33 & 228 & 42,3 \\
Escolaridade & & $13,04(0,791)$ & $13,2(0,746)$ \\
& Ga série & 34 & 33 & 104 & 19,3 \\
& 7a série & 45 & 43,7 & 273 & 50,6 \\
Status socioeconômico & ga série & 24 & 23,3 & 162 & 30,1 \\
& A1 & 3 & 2,9 & 2 & 0,4 \\
& A2 & 10 & 9,7 & 54 & 10 \\
& B1 & 17 & 16,5 & 148 & 27,5 \\
& B2 & 40 & 38,8 & 194 & 36 \\
& C & 32 & 31,1 & 137 & 25,4 \\
& D & 1 & 1 & 4 & 0,7 \\
\hline
\end{tabular}

Instrumentos

Inventário de Habilidades Sociais Para Adolescentes (IHSA-DelPrette, 2009). É um instrumento de autorrelato, referenciado à norma, destinado à população adolescente de 12 a 17 anos de idade e permite avaliar o seu repertório de habilidades sociais em um conjunto de situações interpessoais cotidianas, com diferentes interlocutores (familiares, desconhecidos, pares, autoridades, parceiros afetivos-sexuais) e em distintos contextos: (família, amizade, escola, namoro, lazer). A avaliação do repertório de habilidades sociais é feita com base em indicadores de frequência e dificuldade. $O$ indicador de frequência pontua o quanto o adolescente se comporta de determinada maneira em uma situação. $O$ indicador de dificuldade pontua o quanto é difícil se comportar da maneira descrita no item e refere-se ao custo subjetivo relatado pelo respondente. $O$ instrumento produz um escore geral e escores em fatores: F1-Empatia, F2-Autocontrole, F3-Civilidade, F4- 
Assertividade, F5-Abordagem Afetiva e F6-Desenvoltura Social. Na pesquisa de validação do instrumento (Del Prette \& Del Prette, 2009), os índices de consistência interna do instrumento (coeficiente alpha de Cronbach), nos indicadores de frequência foram: Escore total=0,89; F1 =0,82 F2 =0,72; F3 =0,75; F4 =0,67; F5 =0,69; F6 $=0,61$. Os valores obtidos no indicador dificuldade são: Escore total $=0,90 ; \mathrm{F} 1=0,86 ; \mathrm{F} 2=0,75 ; \mathrm{F} 3=0,83 ; \mathrm{F} 4=0,72 ; \mathrm{F} 5=0,67 ; \mathrm{F} 6$ $=0,51$. $O$ instrumento apresentou indicadores psicométricos aceitáveis também no teste - reteste (Del Prette \& Del Prette, 2009) e é recomendado pelo Conselho Federal de Psicologia.

Inventário de Depressão Infantil (Children Depression's InventoryCDI, Kovacs, 1992). Trata-se da versão brasileira de um instrumento produzido nos Estados Unidos e muito empregado em nosso meio para rastrear sintomas depressivos de crianças e adolescentes, de sete a dezessete anos. A adaptação apresentou um Alfa de Cronbach de 0,85 , demonstrando boa consistência interna (Wathier, Dell'Aglio, \& Bandeira, 2008). Cada item deve ser pontuado com 0 (ausência de sintoma), 1 (presença do sintoma) de 2 (sintoma grave), gerando uma pontuação máxima de 54 pontos. No presente estudo, foi utilizado o ponto de corte de 16 pontos a adolescentes de 12 anos e de 19, para a faixa etária de 13 a 17 anos.

Critério de Classificação Econômica Brasil (CCEB, IBOPE/ABEP, 2013). Trata-se de um instrumento que classifica o nível socioeconômico, a partir de uma pontuação baseada em itens que avaliam a posse de bens de consumo duráveis, instrução do chefe da família e outros fatores, como a presença de empregados domésticos. A pontuação obtida distribui as classes sociais em A1, A2, B1, B2, C, D e E (http://www.abep.org/novo/Content.aspx?ContentID=301, recuperado em 20, maio, 2009).

Procedimento de coleta de dados

A pesquisa foi aprovada pelo Comitê de Ética da Universidade Federal de São Carlos (CEP/UFSCar), sob o parecer de n0144/2009231120011/2009-24 e em cumprimento às normas éticas do Conselho Nacional de Saúde (Resolução 196/96). Após o consentimento da Secretaria Municipal de Educação e da administração das escolas públicas municipais, a primeira autora entrou em contato com os adolescentes e por meio dos quais obteve de seus pais a assinatura do Termo de Consentimento Livre e Esclarecido. A pesquisadora explicou aos adolescentes que o objetivo da pesquisa era conhecer seus pensamentos, sentimentos e comportamentos sociais. Após a entrega do TCLE assinado pelos pais e/ou responsáveis, a coleta ocorreu nas salas de aula, de forma 
coletiva, conduzida pela pesquisadora e na presença do professor de cada sala. Os adolescentes responderam sozinhos aos instrumentos, em um tempo médio de 45 minutos.

Análise dos dados

Primeiramente, foi realizada a análise dos missings tratados pelo método E.M. (expectation-maximization). Posteriormente, foram avaliados os índices de consistência interna (coeficiente alpha de Cronbach) do IHSA-Del-Prette para a amostra deste estudo, nos indicadores de frequência e dificuldade. Os valores obtidos no indicador de frequência foram: Escore total=0,91; $\mathrm{F} 1=0,82$; $\mathrm{F} 2=0,74 ; \mathrm{F} 3=0,79 ; \mathrm{F} 4=0,71 ; \mathrm{F} 5=0,70 ; \mathrm{F} 6=0,63$. Os valores obtidos no indicador dificuldade foram: Escore total=0,93; $F 1=0,87$; $F 2=0,76 ; F 3=0,87 ; F 4=0,79 ; F 5=0,65 ; F 6=0,51$. Em relação ao CDI também foi realizada a análise de consistência interna, com base no coeficiente Alfa de Cronbach e foi encontrado um valor de 0,81 . Para investigar possíveis fatores de risco e proteção entre as habilidades sociais (IHSA-Del-Prette) e variáveis sociodemográficas (ambos tomados como variáveis independentes) sobre os indicadores de depressão (CDI, variável dependente), utilizou-se a análise regressão logística múltipla, no programa SAS 9.0 (SAS Inst., Cary, Estados Unidos), pelo comando procedure logistic. A pontuação total obtida na escala CDI permitiu separar a amostra em dois grupos: adolescentes com (subclínico) e sem (não clínico) indicadores de depressão (variável dicotômica). Dessa maneira, o grupo subclínico assumiu valor 1 (sucesso) e o grupo não clínico assumiu valor 0 (insucesso).

As variáveis independentes possivelmente associadas à indicação de depressão foram os fatores de habilidades sociais do IHSA-Del-Prette, tanto nos indicadores de frequência quanto de dificuldade, considerados como variáveis contínuas: empatia, autocontrole, civilidade, assertividade, abordagem afetiva e desenvoltura social. As variáveis sociodemográficas foram sexo e idade, consideradas variáveis dummy, e o status socioeconômico, considerado como variável contínua.

Precedendo às análises, foi avaliada a presença de multicolinearidade (VIF - Variance Inflation Factor). A adequação do modelo ajustado foi avaliada pelo teste de Hosmer-Lemeshow e a medida da capacidade do modelo discriminar os participantes com indicação de depressão (grupo subclínico), comparado com os participantes sem indicação de depressão (grupo não clínico) foi avaliada pela área sob a curva ROC, de especificidade e sensibilidade. A especificidade refere-se à probabilidade de um indivíduo ser classificado como saudável (insucesso), quando de fato o é. A sensibilidade refere-se à 
probabilidade de um indivíduo ser classificado como doente (sucesso), quando realmente está doente.

Antes de inserir as variáveis no modelo, foram realizadas análises individuais para observar a significância estatística das variáveis independentes em relação à dependente. Todas as variáveis apresentaram relações significativas com os indicadores de depressão, com exceção do fator Desenvoltura Social e do status socioeconômico. Decidiu-se incluir todas as variáveis no modelo, pois, segundo Hosmer e Lemeshow (2000), tal como sugerem vários epidemiologistas com interesse metodológico, com intuito de eliminar variáveis de confusão (uma variável que está associada tanto com a variável resposta quanto com a variável independente).

Posteriormente, as variáveis que apresentaram associação estatisticamente significativa (em torno de $20 \%, p<0,20$ ) com os indicadores de depressão, foram incluídas nas análises multivariadas subsequentes. Odds Ratios (OR) foram estimados a partir da regressão logística múltipla. Segundo Bland e Altman (2000), odds é uma razão entre probabilidades, isto é, a probabilidade do evento ocorrer dividida pela probabilidade do evento não ocorrer. Os odds ratios foram utilizados para avaliar os fatores de risco e de proteção associados à indicação de depressão. Quando os valores estão abaixo da unidade (um; 1), a variável é fator de proteção e quando os valores estão acima da unidade (um; 1 ), a variável é fator de risco.

\section{Resultados}

Primeiramente verificou-se que as variáveis não apresentaram multicolinearidade significativa quando mensurado pelo VIF (Variance Inflation Factor), uma vez que nenhuma delas apresentou valores superiores a 0,90 (Marôco, 2011). A Tabela 2 apresenta a estimativa do modelo das variáveis independentes e o odds ratios ajustado.

Tabela 2. Estimativas do modelo ajustado para variáveis independentes (VI) e odds ratios ajustados. 
Josiane Rosa Campos, Almir Del Prette, Zilda Aparecida Pereira Del Prette Depressão na adolescência: habilidades sociais e variáveis sociodemográficas como fatores de risco/proteção

\begin{tabular}{lcccccc}
\hline \multicolumn{1}{c}{ VI } & Estimativa & Erro Padrão & OR & (IC95)\% & Wald $\boldsymbol{X} \mathbf{2}$ & P-valor \\
\hline Intercept & -0.6943 & 0.5885 & & & 1.3917 & 0.2381 \\
Sexo feminino & 1.4399 & 0.2658 & 4.220 & $2.507-7.105$ & 29.3529 & .0001 \\
Idade 12 anos & -0.7762 & 0.3041 & 0.460 & $0.254-0.835$ & 6.5149 & 0.0107 \\
Idade 14 anos & -0.3638 & 0.2750 & 0.695 & $0.405-1.191$ & 1.7509 & 0.1858 \\
Empatia (F) & -0.0323 & 0.0158 & 0.968 & $0.939-0.999$ & 4.1574 & 0.0415 \\
Autocontrole (F) & -0.0618 & 0.0202 & 0.940 & $0.904-0.978$ & 9.3770 & 0.0022 \\
Civilidade (D) & 0.1003 & 0.0298 & 1.105 & $1.043-1.172$ & 11.3629 & 0.0007 \\
Assertividade (D) & -0.0402 & 0.0301 & 0.961 & $0.906-1.019$ & 1.7869 & 0.1813 \\
Abordagem Afetiva(D) & 0.0521 & 0.0286 & 1.053 & $0.996-1.114$ & 3.3220 & 0.0684 \\
\hline
\end{tabular}

Como se observa na Tabela 2, a análise de regressão múltipla identificou cinco variáveis associadas à indicação de depressão. Observando os valores de OR (odds ratio) e seus intervalos de confiança (de $95 \%$ ), nota-se que a variável sexo feminino (OR= 4.220 ; IC95\% $=2,507-7,105)$ e o fator civilidade no indicador de dificuldade (OR $=1.105$; IC95\% $=1,043 ; 1,172)$ são fatores de risco, uma vez que quanto maior a pontuação nesta variável maior a chance de apresentar indicadores de depressão. A variável idade de 12 anos (OR=0,460; IC95\% $=0,254-0,835)$, bem como as habilidades de empatia (OR=0.968; IC95\%=0.939-0.999) e de autocontrole $(\mathrm{OR}=0,940$; IC95\%=0,904-0,978), nos indicadores de frequência, são fatores de proteção, uma vez que quanto maior a pontuação nesta variável menor a chance de apresentar indicação à depressão.

O modelo final estimado se mostrou adequado de acordo com a estatística de Hosmer-Lemeshow $\left(X^{2}=9.8814 ; p=0,27\right)$, e a medida da capacidade do modelo discriminar os participantes com a característica de interesse (com indicação de depressão) versus participantes sem a característica de interesse (sem indicação de depressão), avaliada pela área sob a curva ROC foi $c=0,77$ que, segundo Hosmer e Lemeshow (2000), apresenta poder discriminante aceitável. $\mathrm{Na}$ Tabela 3, segue-se o percentual de indivíduos identificados corretamente pelo modelo, com os valores de especificidade e sensibilidade.

Tabela 3. Percentual de indivíduos identificados corretamente pelo modelo. 


\begin{tabular}{lccc}
\hline \multirow{2}{*}{ Observado } & \multicolumn{3}{c}{ Predito } \\
\cline { 2 - 4 } & Não clínico & Subclínico & Percentual Correto \\
\hline Grupo não clinico & 531 & 8 & 98,5 \\
Grupo subclínico & 89 & 14 & 13,6 \\
Percentual G lobal & & & $\mathbf{8 4 , 9}$ \\
\hline
\end{tabular}

Como se observa na Tabela 3, o modelo classifica corretamente $98,5 \%$ os participantes classificados como saudáveis (insucesso), apresentando, portanto, alta especificidade; por outro lado, o modelo classifica corretamente apenas $13,6 \%$ os participantes classificados com indicadores de depressão (sucesso), apresentando baixa sensibilidade e, portanto, baixo poder preditivo (Marôco, 2011).

\section{Discussão}

O presente estudo buscou identificar classes específicas de habilidades sociais e variáveis sociodemográficas que poderiam ser fatores de risco ou proteção da depressão na fase inicial da adolescência e se seriam preditivas desse transtorno, com base em indicadores de depressão. Foram inseridas no modelo seis classes de habilidades sociais: empatia, autocontrole, civilidade, assertividade, abordagem afetiva e desenvoltura social, nos indicadores de frequência e dificuldade e as variáveis sociodemográficas idade, sexo e status socioeconômico.

Utilizando a análise de regressão logística múltipla, verificou-se como fatores de proteção a frequência de habilidades de empatia e autocontrole e uma idade mais nova (12 anos em relação a 13 ou 14) e, como fatores de risco, a dificuldade relatada em relação a habilidades de civilidade e ser do sexo feminino. Constatou-se que o poder preditivo do modelo (curva ROC) foi aceitável, embora com alta especificidade e baixa sensibilidade (Marôco, 2011). Em outras palavras, o modelo classificou corretamente $98,5 \%$ dos participantes como saudáveis, ou seja, alta especificidade em excluir esses indivíduos do grupo com indicador de depressão, mas classificou corretamente apenas $13,6 \%$ dos participantes com indicadores de depressão, ou seja, mostrou baixa sensibilidade em termos de poder preditivo.

Os resultados mostram que algumas classes de habilidades sociais podem ser tomadas como fatores de proteção à depressão na adolescência e que déficits ou dificuldades em apresentá-las pode constituir fator de risco, corroborando com outros estudos (Wardet al., 2010; Ross et al., 2010; Lee et al., 2010; Nilsen et al., 2013). Por outro lado, a baixa sensibilidade do poder preditivo se alinha ao 
estudo de Ross et al. (2010). Tais dados podem sinalizar que, embora os déficits de habilidades sociais estejam associados a indicadores de depressão na adolescência, possivelmente eles não predizem a indicação de depressão. Os dados são, portanto, coerentes com a hipótese de Segrin e Flora (2000), de que as habilidades sociais podem auxiliar no enfrentamento de situações estressantes, porém, os antecedentes estressantes podem apresentar maior peso preditivo.

Em relação à classe empatia como fator de proteção, é possível que os adolescentes com maior frequência de comportamento empático criem condições mais favoráveis às relações de amizade e intimidade, estabelecendo maior rede de apoio, o que facilita a obtenção de ajuda e afeto e, consequentemente, proteção contra sintomas depressivos e perda de reforçadores. Por outro lado, a baixa frequência de comportamento empático, como sinaliza o estudo de Schreiter, Pijnenborga, e Ro (2013) com a população adulta, pode sinalizar mal estar e presença de sintomas depressivos. Em concordância com os autores, possivelmente, esses indivíduos estariam mais sob controle de seus próprios sentimentos/pensamentos negativos e dolorosos que sob controle das pessoas ao seu redor, ou de preocupação com o outro.

Referente à frequência de habilidades de autocontrole, como fator de proteção, algumas considerações são necessárias. A literatura aponta que, frequentemente, eventos estressantes aumentam a probabilidade de desenvolvimento de transtornos depressivos (Hammen, 2009). Apresentar comportamentos de autocontrole significa também uma aprendizagem de exposição e tolerância a sentimentos negativos (tristeza, raiva, frustração) e, possivelmente, escolha de cursos de ações menos danosos à saúde. Esta questão pode ajudar a esclarecer, por exemplo, o que Kazdin (1993) aponta em relação à existência de condições de risco na adolescência e ao papel das estratégias de enfrentamento enquanto proteção ao desenvolvimento de transtornos psicológicos.

Mesmo reconhecendo-se que um repertório pobre de habilidades de autocontrole não é a única variável associada aos transtornos depressivos na adolescência, parece importante inseri-lo no planejamento de programas de prevenção e promoção de saúde junto aos adolescentes, à família, à escola, especialmente, no contexto onde foi realizada a pesquisa, como preconiza a WHO (2012). Alguns estudos de intervenção realizados com adolescentes sustentam essa análise e apontam eficácia de desenvolver o repertório de autocontrole, como um dos componentes do tratamento, que resultou em redução dos sintomas depressivos (Stark, Brookman, \& Frazier, 1990; Lewinsohn, Clarke, Hops \& Andrews, 1990) bem como para a importância do autocontrole em outros programas de intervenção voltada para a saúde do 
adolescente (Etscheidt, 1991; Eddy, Reid, \& Fetrow, 2000). Entendese que o ensino de estratégias de autocontrole contribui para o autoconhecimento das emoções, dos sentimentos e do próprio padrão comportamental operante frente às situações consideradas possivelmente estressantes.

Quanto à dificuldade em habilidades sociais de civilidade (no indicador de dificuldade) como um fator de risco, outras considerações são necessárias. O relato de sentir dificuldade em mostrar-se gentil e atender às normas de convívio social parece ser comum em pessoas que apresentam indicadores de depressão (Lejuez et al., 2011; Dougher \& Hackbert, 1994/2003). Essa dificuldade pode ser função de um repertório pobre, por falhas no controle discriminativo e de intensidade da resposta (Boas et al., 2012). O relato pode também, segundo Dougher e Hackbert (1994/2003), estar sob controle de variáveis motivadoras, como a perda de prazer e de função reforçadora dos eventos de vida. Além de uma análise ontogenética, é importante uma análise cultural em relação às habilidades de civilidade. Ferriss (2002) alerta para os modelos de comportamento de não civilidade oferecidos pela mídia moderna, como forma sensacionalista de divulgar e impactar notícias, que podem trazer consequências muito negativas à formação de padrões comportamentais nos indivíduos, incitando a violência. Em outra análise cultural, Sherman (2005) aponta que a cultura atual estimula o jovem à satisfação imediata dos seus desejos, o que pode levar a comportamentos não desejáveis.

No contexto brasileiro, apresentar-se a um grupo de pessoas, cumprimentar os outros e despedir-se, parecem ser habilidades críticas de socialização, como revela o presente estudo. Dessa maneira, a baixa frequência dessa classe de habilidades sociais pode favorecer a rejeição pelos colegas e aumentar a probabilidade de ser excluído de grupos, gerando sentimentos de baixa autoestima. 0 dado parece esclarecer, de alguma maneira, o que a literatura aponta sobre as dificuldades dos adolescentes deprimidos em iniciar e manter relacionamentos interpessoais (Garber, 2006; Nolan et al., 2003) e o isolamento característico das pessoas deprimidas (APA, 2005; Rudolph, 2009). Nesse sentido, parece válido o investimento em programas de prevenção, promoção ou tratamento que focalize não apenas as habilidades de civilidade, mas a classe geral de comunicação de modo a ampliar o repertório social do adolescente. Conforme Del Prette e Del Prette (2005), as habilidades de comunicação são importantes também como pré-requisitos para outras, mais complexas, como as de assertividade, por exemplo.

Quanto às variáveis sociodemográficas, a idade de 12 anos apareceu como fator de proteção (em relação a 13 ou 14 anos) e ser do sexo feminino, como fator de risco. Adolescentes mais novos apresentam menor probabilidade de desenvolver transtornos depressivos, 
portanto, menor idade como fator protetivo era esperada e, é consistente com a literatura (Méndez et al., 2005; Rudolph, 2009; Galambos et al., 2004). Da mesma forma, a vulnerabilidade feminina para a depressão é bem documentada na literatura (APA, 2005; Garber, 2006). Como ocorrem em outros transtornos na infância e na adolescência, as duas variáveis, sexo e idade, podem estar relacionadas (Marsh \& Grasham, 2005).

Contrariamente às expectativas e em concordância com o estudo de Avanci et al. (2008), a variável status socioeconômico nesta amostra não apresentou associação com os indicadores de depressão. Isso pode ter ocorrido porque os dois grupos apresentavam características amostrais bastante homogêneas. Por outro lado, no estudo de Avanci et al. (2008), as características amostrais eram heterogêneas e também não houve predição.

Os dados encontrados não podem ser comparados, em toda sua extensão, a outros estudos, considerando, principalmente os objetivos da investigação. Dessa maneira, o presente estudo parece contribuir, mais no contexto brasileiro, no sentido de identificar quais classes de habilidades sociais seriam potenciais fatores de proteção e de risco à depressão na fase inicial da adolescência, conjuntamente às variáveis sociodemográficas dessa população e no sentido de situar o poder preditivo do modelo.

O presente estudo apresenta algumas limitações. Uma delas foi a não inserção de outras variáveis, além das habilidades sociais do adolescente, como por exemplo, suporte social, desempenho acadêmico, problemas de saúde, eventos estressantes frequentes (Baptista, 1999), o que possivelmente tornaria o modelo mais completo. Tal inserção implicaria no uso de métodos estatísticos mais sofisticados, como o Path Analysis, que poderia ser explorado em futuros estudos, no contexto brasileiro. De uma maneira geral, embora os achados desse trabalho possam sugerir alguns fatores de risco e proteção da depressão na adolescência, pertinentes à socialização, os autores reconhecem a complexidade do fenômeno depressão e a necessidade de ampliar a investigação para os inúmeros determinantes envolvidos nesses transtornos, especialmente na fase inicial da adolescência.

\section{Referências}

American Psychiatric Association (APA, 2005). Diagnostic and Statistical Manual of Mental Disorders, 4a. ed. (DSM-IV). Washington, D. C.

Avanci, J. Q., Assis, S. G., \& Oliveira, R. V. C (2008). Sintomas depressivos na adolescência: Estudo sobre fatores psicossociais 
em amostra de escolares de um município do Rio de Janeiro, Brasil. Caderno de Saúde Pública, 24(10), 2334-2346.

Bahls, S. C. (2002). Epidemiology of depressive symptoms in adolescents of a public school in Curitiba, Brazil. Revista Brasileira de Psiquiatria, 24, 63-67.

Baptista, M. N. (1999). Fatores de risco na depressão. In: M.N Baptista \& F.B Assumpção Júnior (Orgs). Depressão na Adolescência: Uma visão multifatorial. (pp. 31-40). São Paulo: EPU.

Bland, J. M., \& Altman, D. G. (2000). The odds ratio. Education and debate, 320, 1468.

Boas, D. L. O. V., Banaco, R. A., \& Borges, N. B. (2012). Discussões da análise do comportamento acerca dos transtornos psiquiátricos. In N. B. Borges, F. A. Cassas. Clínica analíticocomportamental: Aspectos teóricos e práticos (pp. 95-101). Porto Alegre: Artmed.

Campos, J. R., Del Prette, A., \& Del Prette, Z. A. P. (no prelo). Habilidades sociais e depressão na adolescência: Uma revisão da literatura. Acta Comportamentalia.

Davila, J. (2008). Depressive symptoms and adolescent romance: Theory, research, and implications. Child Development Perspectives, 2(1), 26-31.

Del Prette, A., \& Del Prette, Z. A. P. (2009). Inventário de habilidades sociais para adolescentes (IHSA-Del Prette): Manual de aplicação, apuração e aplicação. São Paulo: Casa do Psicólogo.

Del Prette, Z. A. P., \& Del Prette, A. (2001). Psicologia das habilidades sociais: Terapia e educação. Petrópolis: Vozes.

Del Prette, Z. A. P., \& Del Prette, A. (2010/2012). Social skills and behavior analysis: Historical proximity and new issues. Perspectivas em Análise do Comportamento, 1(2), 104-115 (English version of a Portuguese paper, originally published in 2010).

Del Prette, Z. A. P., Rocha, M. M., Silvares, E. F. M, Del Prette, A. (2012). Social skills and psychological disorders. Universitas Psychologica, 11(3), 941-955.

Dimidjian, S., Barrera, M., Martell, C., Muñoz, R. F., \& Lewinsohn, P. M. (2011). The origins and current status of behavioral activation treatments for depression. Annual Review of Clinical Psychology, 7, 1-38.

Dougher, M. J., \& Hackbert, L. (1994/2003). Uma explicação analítico-comportamental da depressão e o relato de um caso utilizando procedimentos baseados na aceitação. Revista Brasileira Terapia Comportamental Cognitiva, 5(2), 167-184.

Eddy, J. M., Reid, J. B., \& Fetrow, R. A. (2000). An elementary school- based prevention program targeting modifiable antecedents of youth delinquency and violence: Linking the 
Interests of Families and Teachers (LIFT). Journal of Emotional \& Behavioral Disorders, 8(3), 165-176.

Etscheidt, S. (1991). Reducing aggressive behavior and improving self-control: A cognitive-behavioral training program for behaviorally disordered adolescents. Behavioral Disorders, 16(2), 107-115.

Ferriss, A. L. (2002). Studying and measuring civility: A framework, trends, and scale. Sociological Inquiry, 72(3),376392.

Ferster, C. B., Culbertson, S., \& Boren, M. C. P (1977). Princípios do Comportamento. (M. I. R, Silva, Trad.). São Paulo: HUCITEC.

Fortenberry, J. D. (2003). Socioeconomic status, schools, and adolescent depression: Progress in the social epidemiology of adolescent health. The J ournal of Pediatrics, 143(4), 427-429.

Galambos, N. L., Leadbeater, B. J., \& Barker, E. T. (2004). Gender differences in and risk factors for depression in adolescence: A four-year longitudinal study. International Journal of Behavioral Development, 28, 16-25.

Garber, J. (2006). Depression in children and adolescents linking risk research and prevention. American Journal of Preventive Medicine, 31(6), 104-125.

Gresham, F. M., Cook, C. R., Crews, S.D., \& Kern, L. (2004). Social skills training for children and youth with emotional and behavioral disorders. Behavioral Disorders, 30(1), 32-46.

Hammen, C. (2009). Adolescent depression stressful interpersonal contexts and risk for recurrence. Current Directions in Psychological Science, 18(4), 200-204.

Hosmer, D., \& Lemeshow, S. (2000). Applied logistic regression. New York, New York: John Wiley \& Sons.

Instituto Brasileiro de Geografia e Estatística-IBGE (2009). Pesquisa Nacional da Saúde do Escolar-PeNSE. Ministério da Saúde. Rio de Janeiro, RJ.

Kazdin, A. E. (1993). Adolescent mental health: Prevention and treatment programs. American Psychologist, 48(2), 127-141.

Kazdin, A. E., Kraemer, H. C., Kessler, R. C., Kupfer, D. J., \& Offord, D. R. (1997). Contributions of risk-factor research to developmental psychopathology. Clinical Psychology Review, 17, 375-406.

Kosterman, R., Hawkins, J. D., Mason, W. A., Herrenkohl, T. I., Lengua, L. J., \& McCauley, E. (2010). Assessment of behavior problems in childhood and adolescence as predictors of early adult depression. Journal of Psychopathology and Behavioral Assessment, 32(1), 118-127.

Kovacs, M. (1992). The Children's Depression Inventory, CDI Manual Multi-Health Systems, Inc. Toronto. John Seeley 
Lee, A., Hankin, B. L., \& Mermelstein, R. J. (2010). Perceived social competence, negative social interactions and negative cognitive style predict depressive symptoms during adolescence. Journal Clinical Child Adolescent Psychology, 39(5), 603-615.

Lejuez, C. W., Hopko., D. R., Acierno., R., Daughters., S. B., \& Pagoto, S. L. (2011). Year revision of the brief Behavioral Activation treatment for depression: Revised. Treatment Manual Behavior Modification, 35(2) 111-161.

Lewinsohn, P. M., Clarke, G. N., Hops, H. \& Andrews, J. (1990). Cognitive-behavioral treatment for depressed adolescents. Behavior Therapy, 21,385-401.

Marôco, J. (2011). Análise Estatística com o SPSS STATISTICS. Editor: Report Number.

Marsh, E. J., \& Graham, S. A. (2005). Classificação e tratamento da psicopatologia infantil. In: V. E. Caballo \& M. A. Simón (Orgs.). Manual de Psicologia Clínica Infantil e do Adolescente: Transtornos Gerais (pp. 29-56). São Paulo: Livraria Santos.

Méndez, F. X., Olivares, J., \& Ros, M. C. (2005). Características clínicas e tratamento da depressão na infância e adolescência. In: V. E. Caballo \& M. A. Simón (Orgs.), Manual de Psicologia Clínica Infantil e do Adolescente: Transtornos Gerais (pp. 139185). São Paulo: Livraria Santos.

Moraes, A. B. A., \& Rolim, G. S. (2012). Algumas reflexões analíticocomportamentais na área da psicologia da saúde. In $\mathrm{N}$. B. Borges, F. A. Cassas. Clínica analítico-comportamental: Aspectos teóricos e práticos (pp. 287-293). Porto Alegre: Artmed.

Nilsen, W., Karevold, E., Roysamb, E., Gustavson, K., \& Mathiesen, K. S. (2013). Social skills and depressive symptoms across adolescence: Social supports a mediator in girls versus boys. J ournal of Adolescence, 36(1), 11-20.

Nolan, S. A., Flynn, C., \& Garber, J. (2003). Prospective relations between rejection and depression in young adolescents. Journal of Personality and Social Psychology, 85(4), 745-755.

Novak, G., \& Pelaez, M. (2004). Child and adolescent development: A behavioral systems approach. Thousand Oaks (CA): Sage Publications.

Reppold, C. T., \& Hutz, C. S. (2003). Prevalência de indicadores de depressão entre adolescentes no Rio Grande do Sul. Avaliação Psicológica, 2(2), 175-184.

Ross, A. G., Shochet I. M., \& Bellair, R. (2010). The role of social skills and school connectedness in preadolescent depressive symptoms. Journal of Clinical Child \& Adolescent Psychology, $39(2), 269-75$. 
Rudolph, K. D. (2009). Adolescent depression. In: I. H. Gotlib., \& C. L. Hammen (Eds.), Handbook of Depression (pp. 444-466). New York: The Guilford Press.

Schreiter, S., Pijnenborg, G. H. M., \& Rot, M. (2013). Empathy in adults with clinical or subclinical depressive symptoms. Journal of Affective Disorders, 150, 1-16.

Sherman, N. (2005). Of manners and morals. Britsh Journal of Educational Studies, 53(3), 272-289.

Segrin, C (2000). Social skills deficits associated with depression. Clinical Psychology Review, 20(3), 379-403.

Segrin, C., \& Flora, J. (2000). Poor social skills are a vulnerability fator in the development of psychosocial problems. Human Communication Research, 26(3), 489-514.

Soenens, B., Park, S. Y., Vansteenkiste, M., Mouratidis, A. (2012). Perceived parental psychological control and adolescent depressive experiences: A cross-cultural study with Belgian and South-Korean adolescents. Journal of Adolescence, 35(2), 261272.

Stark, K. D., Brookman, C, S., \& Frazier, R. (1990). A comprehensive school-based treatment program for depressed children. School Psychology Quarterly, 5(2), 111-140.

Ward, S., Sylva, J., \& Gresham, F. M. (2010). School-based predictors of early adolescent depression. School Mental Health, 2(3), 125-131.

Wathier, J. L, Dell'aglio, D. D. E., \& Bandeira, D. R. (2008). Análise fatorial do Inventário de Depressão Infantil (CDI) em amostra de jovens brasileiros. Avaliação Psicológica, 7(1), 75-84.

Wight, R. G., Botticello, A. L., \& Aneshensel, C. S. (2006). Socioeconomic context, social support, and adolescent mental health: A multilevel investigation. Journal of Youth and Adolescence, 35(1), 109-120.

World Health Organization (2012). Adolescent mental health: mapping actions of nongovernmental organizations and other international development organizations. Genebra.

\section{Endereço para correspondência \\ J osiane Rosa Campos}

Universidade Federal de São Carlos

Centro de Educação e Ciências Humanas

Programa de Pós-Graduação em Psicologia

Rodovia Washington Luis, Km 235 - Caixa Postal 676, CEP 13565-905, São Carlos $\mathrm{SP}$, Brasil

Endereço eletrônico: josirosacampos@gmail.com

\section{Almir Del Prette}

Universidade Federal de São Carlos

Centro de Educação e Ciências Humanas

Programa de Pós-Graduação em Psicologia 
Josiane Rosa Campos, Almir Del Prette, Zilda Aparecida Pereira Del Prette Depressão na adolescência: habilidades sociais e variáveis sociodemográficas como fatores de risco/proteção

Rodovia Washington Luis, Km 235 - Caixa Postal 676, CEP 13565-905, São Carlos SP, Brasil

Endereço eletrônico: adprette@ufscar.br

\section{Zilda Aparecida Pereira Del Prette}

Universidade Federal de São Carlos

Centro de Educação e Ciências Humanas

Programa de Pós-Graduação em Psicologia

Rodovia Washington Luis, Km 235 - Caixa Postal 676, CEP 13565-905, São Carlos SP, Brasil

Endereço eletrônico: zdprette@ufscar.br

Recebido em: 19/03/2014

Reformulado em: 07/05/2014

Aceito para publicação em: 12/05/2014

\section{Notas}

* Psicóloga, Mestra e Doutoranda em Psicologia pela Universidade Federal de São Carlos.

** Psicólogo, Mestre em Psicologia pela Pontifícia Universidade Católica de Campinas e Doutor em Psicologia (Psicologia Experimental) pela Universidade de São Paulo.

*** Psicóloga, Mestra em Psicologia (Psicologia Social) pela Universidade Federal da Paraíba, Doutora em Psicologia (Psicologia Experimental) pela Universidade de São Paulo e Pós-doutora em Psicologia das Habilidades Sociais pela Universidade da Califórnia.

\section{Agradecimentos}

Os autores agradecem aos participantes e aos diretores das escolas.

Este trabalho foi financiado pela Coordenação de Aperfeiçoamento de Pessoal de Nível Superior (CAPES), com bolsa de doutoramento atribuída à primeira autora. 